\title{
epicentre
}

an Illumina company

\section{Rapid, directional RNA-seq library preparation kits for formalin-fixed paraffin-embedded RNA}

\author{
Preparing RNA-seq libraries using RNA isolated from formalin-fixed paraffin-embedded (FFPE) tissue \\ is notoriously difficult, due to limited sample amounts and RNA degradation. Here we describe the \\ ScriptSeq ${ }^{\text {TM }}$ Complete Kits, which enable researchers to go from as little as $100 \mathrm{ng}$ of total FFPE RNA \\ to directional, cluster-ready RNA-seq libraries in $1 \mathrm{~d}$. The libraries are virtually free of contaminating \\ ribosomal RNA (rRNA) and exhibit high quality scores and percentage of mapped reads.
}

\section{Introduction}

Gene-expression profiling of the millions of currently available FFPE tissue samples using massively parallel RNA-seq could greatly add to our knowledge of cancer biology, biomarker discovery and, ultimately, new drug development. Optimal RNA-seq results are obtained after rRNA depletion to maximize sequencing depth, transcript coverage and the number of uniquely mapped reads. Unfortunately, the highly degraded nature of most FFPE RNA samples precludes using poly $(\mathrm{A})^{+}$ RNA enrichment-the most common method for treating total RNA prior to RNA-seq library preparation.

In this report, we demonstrate that the ScriptSeq Complete Kits offer a seamless, end-to-end solution for preparing RNA-seq libraries in $1 \mathrm{~d}$ even when using highly degraded FFPE RNA samples. The ScriptSeq Complete Kits produce high-quality RNA-seq libraries, as assessed by the low percentage of rRNA reads, the high-quality score metrics (>Q30) (ref. 1,2), the percentage of mapped reads and $>98 \%$ directionality.

\section{ScriptSeq Complete Kit process}

The ScriptSeq Complete Kits are composed of two modules: the Ribo-Zero ${ }^{\mathrm{TM}}$ module, which removes rRNA from the sample, and the ScriptSeq v2 module, which produces cluster-ready libraries from the Ribo-Zero-treated sample. An overview of the single-day ScriptSeq Complete process is depicted in Figure 1.

The Ribo-Zero module is specific to each ScriptSeq Complete Kit. For example, the ScriptSeq Complete Kit includes Ribo-Zero reagents that remove the cytoplasmic 28S, 18S, 5.8S and 5S rRNA from human, mouse or rat total RNA samples. The ScriptSeq Complete Gold Kit includes Ribo-Zero Gold reagents that remove cytoplasmic rRNAs as well as the $16 \mathrm{~S}$ and $12 \mathrm{~S}$ mitochondrial rRNAs.

\section{Jim Pease \& Roy Sooknanan}

Epicentre (an Illumina company), Madison, Wisconsin, USA. Correspondence should be addressed to J.P. (jim.pease@epicentre.com).
The ScriptSeq v2 module uses random-primed cDNA synthesis and a unique, patented terminal-tagging reaction to prepare directional libraries in under $4 \mathrm{~h}$. If desired, Illumina ${ }^{\circledR}$ Index reads can be readily incorporated into the libraries.

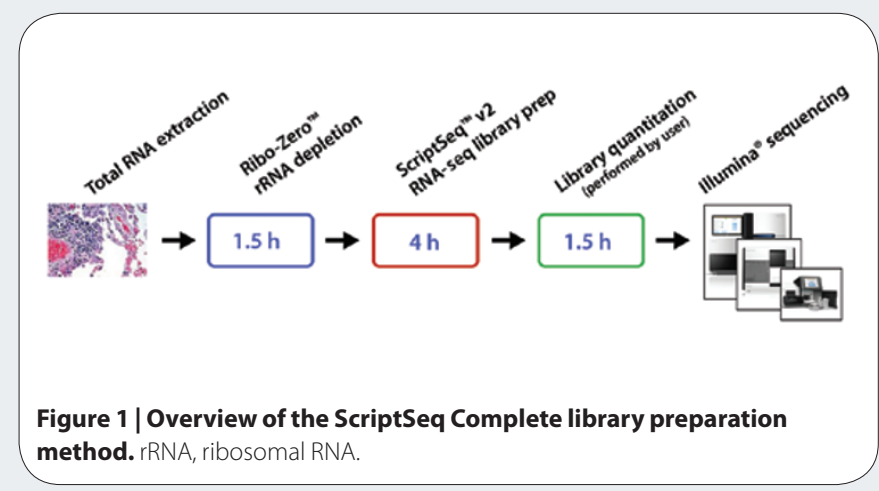

RNA-seq library preparation from FFPE tissue

We prepared ScriptSeq Complete libraries from two human FFPE RNA samples (Fig. 2a, severely fragmented; Fig. 2b, less fragmented). From each FFPE RNA sample, 100 ng was treated with either the Ribo-Zero or Ribo-Zero Gold reagents. The entire amount of the Ribo-Zerotreated RNA recovered was used to prepare ScriptSeq v2 libraries. The libraries were sequenced using an Illumina GAllx sequencer, and alignment was performed using the CASAVA 1.7 pipeline and additional in-house scripts.

As shown in Figure 3, the Ribo-Zero reagents reduced the cytoplasmic rRNA contamination in the libraries to less than $4 \%$ of the total sequencing reads for even the most degraded FFPE RNA (sample (A) in Fig. 3). The Ribo-Zero Gold reagents reduced the number of cytoplasmic rRNA reads to less than $5 \%$ and mitochondrial rRNA reads to less than $3 \%$ for the same sample. All libraries exhibited $>92 \%$ of clusters passing filter at $>$ Q30. The libraries also demonstrated $>98 \%$ directional reads. 


\section{APPLICATION NOTES}
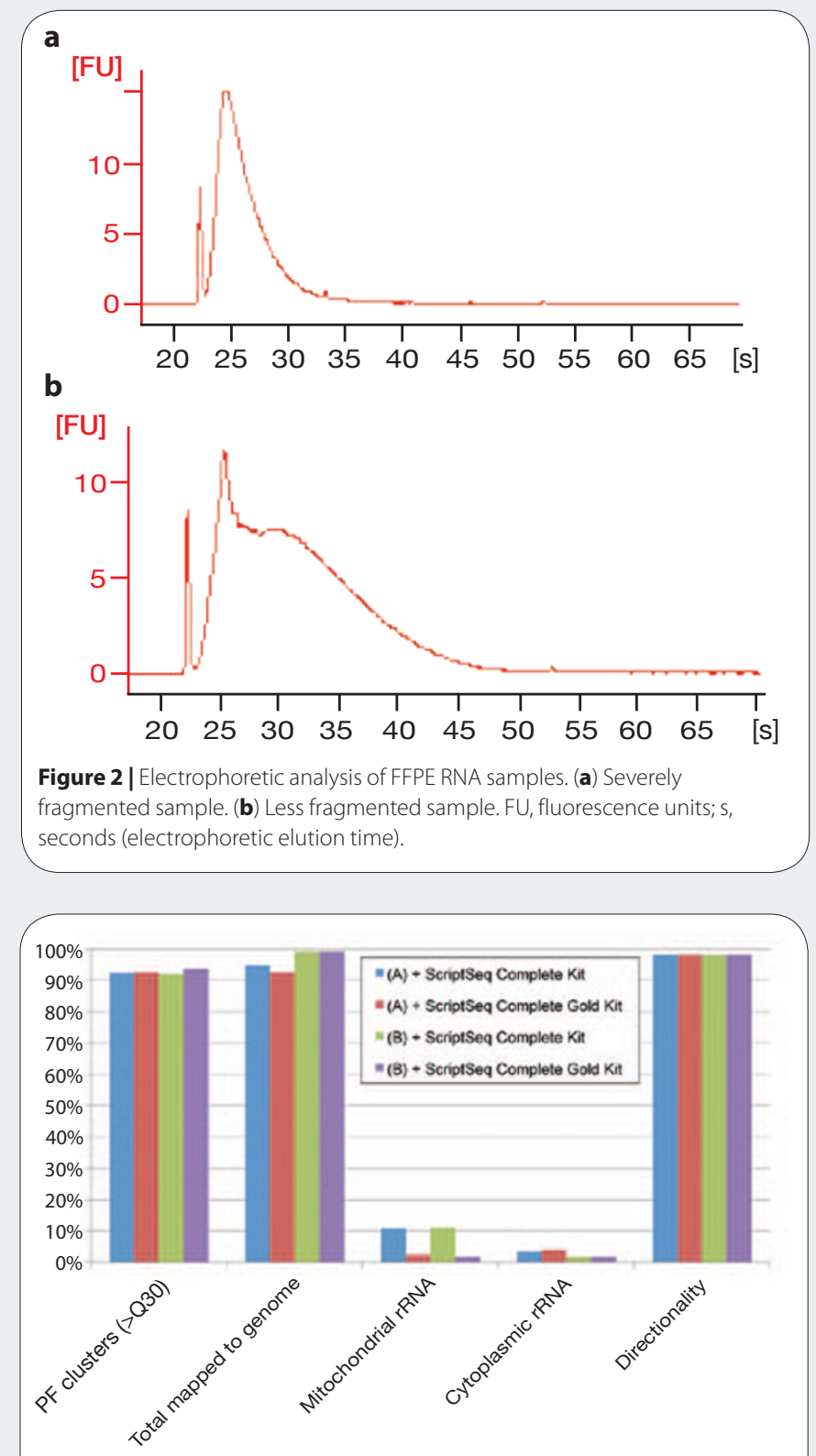

Figure 3 | ScriptSeq Complete library metrics from two formalin-fixed paraffin-embedded RNA samples. Two samples ((A) and (B)) were treated with both the standard Ribo-Zero module and the Ribo-Zero Gold module for ribosomal RNA (rRNA) removal.

\section{Conclusions}

The ScriptSeq Complete Kits enable researchers to go from FFPE total RNA samples to cluster-ready RNA-seq libraries in less than $1 \mathrm{~d}$. The Ribo-Zero modules of the kits remove $>95 \%$ of rRNA from the samples. ScriptSeq Complete Kits produce high-quality RNA-seq libraries from FFPE RNA as judged by standard sequencing metrics.

\section{ACKNOWLEDGEMENTS}

We are grateful to John Hitchen (Epicentre) and Brad Baas (Illumina) for technical assistance and Cristine Kinross (Epicentre) for assistance with data analysis.

1. Ewing, B. et al. Base-calling of automated sequencer traces using phred. I. Accuracy assessment. Genome Res. 8, 175-185 (1998).

2. Ewing, B. \& Green, P. Base-calling of automated sequencer traces using phred. II. Error probabilities. Genome Res. 8, 186-194 (1998). 